in standard than a London B.A. : on the other hand, many Nigerians are impatient that, in a country which needs graduates urgently, so few students graduate from Ibadan and such high standards of entry are demanded. This is one example of the massive unsolved problems which accompany the experiment of exporting the University of London to the African bush. Dr. Mellanby's book is a welcome record of the sincerity and enthusiasm of those who took part in founding Nigeria's university, and the sustained help which the University of London has given to the cause of higher education in the Colonies.

E. Ashby

\section{FROM CONDUCTORS TO TRANSISTORS}

Electric Conduction in Semiconductors and Meta's By Dr. W. Ehrenberg. Pp. $x+390$. (Oxford: Clarendon Press; London: Oxford University Press, 1958.) 63s. net.

$\mathrm{T}$ HE scope of work on semiconductors has increased in recent years to such an extent that it is no longer practicable for one book to cover the whole field in detail. Dr. Ehrenberg has therefore wisely confined his attention to transport properties, leaving on one side studies of magnetic susceptibilities and surface adsorption (for example), and giving only little space to questions of absorption and emission of infra-red radiation and of X-rays (5 and 3 pages, respectively). If this restriction is granted, it is convenient to treat the subject under the following broad headings.

First, the theoretical basis of band theory and its experimental checks are discussed. This is fundamental for both equilibrium and transport properties. The author spends Chapters 5 and 6 on this topic (65 pages), half the space being devoted to the wavemechanical problem. He gives a clear introduction which should enable the reader to pass readily to more advanced discussions. This applies in particular to the author's introduction to Bloch's theorem, which he makes plausible in a very simple manner. It also applies to the concept of Brillouin zones, which are introduced by reference to the simple cubic lattice, rather than by more general arguments.

Secondly, an account is given of electron scattering and times of relaxation. Whereas for band theory one could refer the reader to existing standard texts (though, strangely, this is never done in books on semiconductors), this second topic is characteristic of transport phenomena, since it includes the origin of the electrical resistance of materials. The author treats this subject on pp. 55-63 and in Chapters 7 and 8 (a total of 51 pages). A full account of lattice scattering and of the original treatment of ionized impurity scattering is given here, but neutral impurity scattering receives only a brief mention (p. 244).

Thirdly, armed with this theoretical equipment, the author derives more or less formal expressions for the transport coefficients (conductivity, magneto-resistive ratio, Hall and thermo-electric coefficients, etc.), under various conditions of scattering (Chapters 4 and 9, 35 pages). These may then be compared with experiment (Chapter 10, 54 pages). It is good to see that the author has here not restricted himself to germanium and silicon, but has gone on to discuss the older low-mobility semiconductors, on some of which new work is badly needed. On the other hand, results for metals are scarcely discussed, and topics such as the skin effect in metals are also not treated, so that the term 'metals' in the title could be misleading.

The reader should be warned that some of the results in Chapter 4 appear in unfamiliar guise. The reason for this is that the author gives simple but approximate expressions for the Fermi integrals (p. 38) :

$$
\int_{0}^{\infty} \frac{x^{n} d x}{1+\lambda \exp x} \sim \frac{a_{n}}{\lambda+b_{n}}
$$

where $a_{n}, b_{n}$ are numbers, and sometimes uses these in the standard results. The numerical information concerning the $a$ 's and $b$ 's is new, and, as an error of less than 5 per cent is claimed for $\lambda>1 / 6$, it may often be useful.

Fourthly, the author discusses the non-linear properties of semiconductors which have led to their applications as circuit elements, and which are largely responsible for the widespread interest in the whole field. Dr. Ehrenberg has here made a serious effort to give an account of these developments, and has devoted more than a quarter of the book to them (108 pages). He discusses barrier layers and rectification, excess and minority carriers, their life-times, and various types and aspects of transistors. But space considerations clearly did not allow him to discuss the more complicated flow and space charge regimes in transistors.

Because the book is so broadly based, and because the author has read so widely, this is a most useful book which beginners as well as workers in this field will want to possess. The beginner should note that he has not really wasted his time when, after diligent study to p. 33 , he reads "The absurd result obtained here indicates therefore a serious misapplication of the model" or when doubt is again thrown (on p. 81) on the preceding work. It might have been simpler if the author had started with what he regards as a satisfactory model (Chapter 5), and if he had introduced those results of Chapters 2-4 which are in fact needed at the appropriate points in the discussion of this model. This would have obviated the two revisions of the model in the first quarter of the book.

Although the preface is dated January 1958, internal evidence suggests that the manuscript itself was completed by 1956 . When a subject develops as rapidly as the one here under review, and when the author has evaluated a great deal of recent information, a delay of two years before publication is achieved must be a matter of regret. In spite of this handicap, this book is one of the most balanced and thorough accounts of this field which is at present available.

P. T. Land SBera

\section{PROGRESS IN PHYSICS}

Reports on Progress in Physics

Vol. 20 (1957). Pp. iv +568. Vol. 21 (1958). Pp. iv + 383. Edited by A. C. Stickland. (London: The Physical Society, 1957, 1958.) Each 63s. net.

WTIH the increasing pace of scientific research, the collection of information regarding recent advances in any specialized branch of investigation is a formidable task and the demand has therefore grown for detailed progress reports and review articles. 